\title{
BAYESIAN MODEL UPDATING OF STOCHASTIC DYNAMIC SOIL-STRUCTURE INTERACTION SYSTEMS WITH MULTIPLE RESOLUTIONS
}

\author{
Sahil Bansal ${ }^{1}$, Sai Hung Cheung ${ }^{1}$ \\ ${ }^{1}$ School of Civil and Environmental Engineering, Nanyang Technological University, Singa- \\ pore (shcheung@ntu.edu.sg)
}

\begin{abstract}
In this paper, the problem of stochastic modeling of dynamic soil-structure interaction systems and the corresponding model updating problem are considered. Ambient vibration or seismic vibration data can be used to update the model properties of dynamic soilstructure interaction systems. Most existing works for model updating of these types of systems consider only one choice of resolution without paying much attention to the effect of model resolution. In this paper, a Bayesian model updating methodology based on stochastic model classes with multiple resolutions is proposed. The effect of model resolution on the result of the Bayesian model updating will be shown. The proposed methods are illustrated with an example involving a dynamic soil-building-structure system.
\end{abstract}

Keywords: Bayesian, Stochastic, Model updating, multiple resolutions, Soil-structure interaction system.

\section{INTRODUCTION}

The problem of model updating using dynamic response data has received much attention over the years because of its wide range of application in structural health monitoring, structural response prediction, reliability and risk assessment, and structural control. The need for model updating arises because there are always modeling errors and uncertainties associated with the process of constructing a mathematical model of a system. Once a mathematical model is specified, the problem reduces to estimation of model parameters that improves the agreement between the predicted and actual response. However, no single deterministic input-output model can make perfect predictions about the dynamic characteristics of a system and its prediction will always be uncertain. Therefore, there has been increasing interest in using Bayesian statistical framework for updating structural model based on dynamic response data [1-5]. Bayesian model updating methodology not only identifies the optimal system model but a set of plausible models, quantified by a probability distribution over model parameters that describe the complete picture of the uncertainty [1]. It also allows for the explicit treatment of the ill-conditioning and non-uniqueness arising in the model updating inverse problem. 
Most works in Bayesian finite-element model updating only focus on how to match the model predicted response with the measured response without paying too much attention to the effect of model resolution (in space and time) to the uncertainty quantification. It is well known that the accuracy of the transient dynamic solution depends on the discretization of the model: the smaller the time step, the higher is the accuracy. Similarly, the choice of discretization scheme employed in spatial domain is important in the numerical modeling of wave propagation (especially for the soil domain, in soil-structure interaction system). Reducing the spatial resolution may decrease the computational effort requirement but can bring undesirable impacts to the analysis results. In general, spatial discretization scheme is based upon the concept of resolving the propagation of the shear waves at or below a particular frequency by ensuring that an adequate number of elements fit within the wavelength of the chosen shear wave. This ensures that the mesh is refined enough such that the desired aspects of the propagating waves are well captured in the analysis.

The focus of this paper is to investigate the effects of choice of spatial discretization scheme employed on the posterior distribution of uncertain parameters during the process of Bayesian model updating. In the following section, the formulation of the Bayesian methodology for model updating is presented. The proposed method is illustrated with an example involving a dynamic soil-building-structure system.

\section{STOCHASTIC SYSTEM MODEL CLASS UPDATING}

\subsection{Stochastic System Model Class}

Consider a set of possible models specified by a model class $\mathcal{M}$, describing the inputoutput behavior of a system [1]. $\mathcal{M}$ is parameterized by parameter vector $\boldsymbol{\theta} \in \mathbb{R}^{D}$ and the initial relative plausibility of each predictive model $\mathcal{M}(\boldsymbol{\theta})$ is specified by an initial probability distribution function (PDF) $p(\boldsymbol{\theta} \mid \mathcal{M})$, called the prior. By Bayes' theorem the posterior PDF $p(\boldsymbol{\theta} \mid \mathcal{D}, \mathcal{M})$, i.e. the updated relative plausibility of each predictive model based on inclusion of measured data is then given by:

$$
p(\boldsymbol{\theta} \mid \mathcal{D}, \mathcal{M})=c^{-1} p(\mathcal{D} \mid \boldsymbol{\theta}, \mathcal{M}) p(\boldsymbol{\theta} \mid \mathcal{M}) .
$$

where $c=p(\mathcal{D} \mid \mathcal{M})$ is the normalizing constant and $p(\mathcal{D} \mid \boldsymbol{\theta}, \mathcal{M})$ is the likelihood function which expresses the probability of getting $\mathcal{D}$ based on the predictive PDF for the response of the model specified by $\boldsymbol{\theta}$.

\subsection{General Formulation for Model Classes}

A stochastic system model class can be constructed from a deterministic state-space model by stochastic embedding [1]. In this process, along with uncertain system parameters, prediction-error terms are included in the equations of motion. The following formulation is obtained or modified from those presented in [6]. The continuous-time state-space model of 
stochastic linear dynamic system is given by a first order stochastic differential equation as follows:

$$
\begin{gathered}
\dot{\mathbf{x}}(t)=\mathbf{A}_{c}(\boldsymbol{\theta}) \mathbf{x}(t)+\mathbf{B}_{c}(\boldsymbol{\theta}) \mathbf{u}(t)+\mathbf{w}(t) \\
\mathbf{y}(t)=\mathbf{C}(\boldsymbol{\theta}) \mathbf{x}(t)+\mathbf{D}(\boldsymbol{\theta}) \mathbf{u}(t)+\mathbf{v}(t) \\
\mathbf{x}(0)=\mathbf{x}_{0} . \\
\mathrm{E}\left[\mathbf{w}(t) \mathbf{w}^{T}(\tau)\right]=\mathbf{Q}_{c}(\boldsymbol{\theta}) \delta(t-\tau) . \\
\mathrm{E}\left[\mathbf{v}(t) \mathbf{v}^{T}(\tau)\right]=\mathbf{R}_{c}(\boldsymbol{\theta}) \delta(t-\tau) .
\end{gathered}
$$

The system matrix $\mathbf{A}_{c}, \mathbf{B}_{c}, \mathbf{C}_{c}$, and $\mathbf{D}_{c}$ are a specified functions of uncertain system parameters ө. $\mathbf{x}(t) \in \mathbb{R}^{N_{x}}, \mathbf{u}(t) \in \mathbb{R}^{N_{u}}, \mathbf{y}(t) \in \mathbb{R}^{N_{y}}$ denote the state, excitation and output measurement vectors at time $t$ and $\mathbf{x}_{0}$ is the initial condition vector. The random variables $\mathbf{w}$ and $\mathbf{v}$ represent the uncertain component of the input (e.g., ambient vibration or white noise input to stochastic ground motion model) and the measurement noise, modeled as independent Gaussian variables, respectively. The corresponding discrete-time state-space model with time step $\Delta t$ (where $t_{n}=n \Delta t$ ), is:

$$
\begin{gathered}
\mathbf{x}_{n}=\mathbf{A}(\boldsymbol{\theta}) \mathbf{x}_{n-1}+\mathbf{B}(\boldsymbol{\theta}) \mathbf{u}_{n-1}+\mathbf{w}_{n-1} . \\
\mathbf{y}_{n}=\mathbf{C}(\boldsymbol{\theta}) \mathbf{x}_{n}+\mathbf{D}(\boldsymbol{\theta}) \mathbf{u}_{n}+\mathbf{v}_{n} .
\end{gathered}
$$

where $\mathbf{A}(\boldsymbol{\theta})=\exp \left(\Delta t \mathbf{A}_{c}(\boldsymbol{\theta})\right)$ and $\mathbf{B}(\boldsymbol{\theta})=\mathbf{A}_{c}^{-1}(\boldsymbol{\theta})(\mathrm{I}-\mathbf{A}(\boldsymbol{\theta})) \mathbf{B}_{c}(\boldsymbol{\theta})$. Uncertain input $\mathbf{w}_{n}$ at time $t_{n}$ is modeled by a multivariate Gaussian distribution with covariance matrix $\mathbf{Q}(\boldsymbol{\theta})$, given by [7]:

$$
\mathbf{Q}(\boldsymbol{\theta})=\int_{0}^{\Delta t} \exp \left(\boldsymbol{A}_{c}(\boldsymbol{\theta}) \tau\right) \mathbf{Q}_{c}(\boldsymbol{\theta}) \exp \left(\boldsymbol{A}_{c}(\boldsymbol{\theta}) \tau\right)^{T} d \tau
$$

The uncertain prediction-error term $\mathbf{v}_{n}$, has covariance matrix $\mathbf{R}(\boldsymbol{\theta})$ given by:

$$
\mathbf{R}(\boldsymbol{\theta})=\mathbf{R}_{c}(\boldsymbol{\theta}) / \Delta t
$$

Uncertain input $\mathbf{w}_{n}$ and prediction-error $\mathbf{v}_{n}$, are assumed to be independent of each other at all times. $\mathbf{Q}$ and $\mathbf{R}$ are also specified function of uncertain system parameters $\boldsymbol{\theta}$. Let $\mathbf{X}_{n}=\left[\mathbf{x}_{0}^{T}, \mathbf{x}_{1}^{T}, \ldots, \mathbf{x}_{n}^{T}\right]^{T}$ and $\mathbf{Y}_{n}=\left[\mathbf{y}_{0}^{T}, \mathbf{y}_{1}^{T}, \ldots, \mathbf{y}_{n}^{T}\right]^{T}$. Given $\boldsymbol{\theta}$, the predictive PDF of output $\mathbf{Y}_{N}$ can be written as the product of conditional probabilities as follows:

$$
p\left(\mathbf{Y}_{N} \mid \boldsymbol{\theta}\right)=p\left(\mathbf{y}_{0} \mid \boldsymbol{\theta}\right) \prod_{n=1}^{N} p\left(\mathbf{y}_{n} \mid \mathbf{Y}_{n-1}, \boldsymbol{\theta}\right)
$$

The conditional distribution $p\left(\mathbf{y}_{n} \mid \mathbf{Y}_{n-1}, \boldsymbol{\theta}\right)$ follows a multivariate Gaussian distribution with mean $\mathrm{E}\left[\mathbf{y}_{n} \mid \mathbf{Y}_{n-1}, \boldsymbol{\theta}\right]=\mathbf{y}_{n \mid n-1}$ and covariance matrix $\operatorname{Cov}\left(\mathbf{y}_{n} \mid \mathbf{Y}_{n-1}, \boldsymbol{\theta}\right)=\mathbf{S}_{n \mid n-1}$ : 


$$
p\left(\mathbf{y}_{n} \mid \mathbf{Y}_{n-1}, \boldsymbol{\theta}\right)=\frac{1}{(2 \pi)^{N y / 2}\left|\mathbf{S}_{n \mid n-1}\right|^{1 / 2}} \exp \left(-\frac{1}{2}\left(\mathbf{y}_{n}-\mathbf{y}_{n \mid n-1}\right)^{T} \mathbf{S}_{n \mid n-1}^{-1}\left(\mathbf{y}_{n}-\mathbf{y}_{n \mid n-1}\right)\right)
$$

$\mathbf{y}_{n \mid n-1}$ and $\mathbf{S}_{n \mid n-1}$ can be calculated by the following formulae using the Kalman filter which comes from Bayesian sequential state updating with $\mathbf{x}_{0 \mid-1}=\mathbf{x}_{0}, \mathbf{P}_{0 \mid-1}=\mathbf{I}$ and $\mathbf{S}_{0 \mid-1}=\mathbf{R}$ :

$$
\begin{gathered}
\mathbf{x}_{n \mid n-1}=\mathbf{A}(\boldsymbol{\theta}) \mathbf{x}_{n-1 \mid n-1}+\mathbf{B}(\boldsymbol{\theta}) \mathbf{u}_{n-1} . \\
\mathbf{P}_{n \mid n-1}=\mathbf{A}(\boldsymbol{\theta}) \mathbf{P}_{n-1 \mid n-1} \mathbf{A}(\boldsymbol{\theta})^{T}+\mathbf{Q}(\boldsymbol{\theta}) . \\
\mathbf{y}_{n \mid n-1}=\mathbf{C}(\boldsymbol{\theta}) \mathbf{x}_{n \mid n-1}+\mathbf{D}(\boldsymbol{\theta}) \mathbf{u}_{n-1} . \\
\mathbf{S}_{n \mid n-1}=\mathbf{C}(\boldsymbol{\theta}) \mathbf{P}_{n \mid n-1} \mathbf{C}(\boldsymbol{\theta})^{T}+\mathbf{R}(\boldsymbol{\theta}) . \\
\mathbf{x}_{n \mid n}=\mathbf{x}_{n \mid n-1}+\mathbf{P}_{n \mid n-1} \mathbf{C}(\boldsymbol{\theta})^{T} \mathbf{S}_{n \mid n-1}^{-1}\left(\mathbf{y}_{n}-\mathbf{y}_{n \mid n-1}\right) . \\
\mathbf{P}_{n \mid n}=\mathbf{P}_{n \mid n-1}-\mathbf{P}_{n \mid n-1} \mathbf{C}(\boldsymbol{\theta})^{T} \mathbf{S}_{n \mid n-1}^{-1} \mathbf{C}(\boldsymbol{\theta}) \mathbf{P}_{n \mid n-1} .
\end{gathered}
$$

Thus, $p\left(\mathbf{Y}_{N} \mid \boldsymbol{\theta}\right)$ in (10) is given by:

$$
p\left(\mathbf{Y}_{N} \mid \boldsymbol{\theta}\right)=\frac{1}{(2 \pi)^{N y(N+1) / 2} \prod_{n=0}^{N}\left|\mathbf{S}_{n \mid n-1}\right|^{1 / 2}} \exp \left(-\frac{1}{2} \sum_{n=0}^{N}\left(\mathbf{y}_{n}-\mathbf{y}_{n \mid n-1}\right)^{T} \mathbf{S}_{n \mid n-1}^{-1}\left(\mathbf{y}_{n}-\mathbf{y}_{n \mid n-1}\right)\right)
$$

The posterior PDF of $\boldsymbol{\theta}$ is then given by (1) where $\mathcal{D}=\hat{\mathbf{Y}}_{N}$, the measurement for the system output $\mathbf{Y}_{N}$.

\subsection{Model Class Comparison}

Model class comparison is a rigorous Bayesian updating procedure that judges the plausibility of different candidate model classes based on their posterior probability (their probability conditional on the data from the system). Its application to system identification of dynamic systems that are globally identifiable or unidentifiable was studied in $[2,4,8]$. In these publications, a model class is referred to as a Bayesian model class. Given a set of candidate model classes $\boldsymbol{M}=\left\{\mathcal{M}_{i}: i=1, . ., N_{M}\right\}$, the posterior probability $P\left(\mathcal{M}_{i} \mid \mathcal{D}, \boldsymbol{M}\right)$ of each model class based on system data $\mathcal{D}$ is given by Bayes' theorem as:

$$
P\left(\mathcal{M}_{i} \mid \mathcal{D}, \boldsymbol{M}\right)=\frac{p\left(\mathcal{D} \mid \mathcal{M}_{i}\right) P\left(\mathcal{M}_{i} \mid \boldsymbol{M}\right)}{p(\mathcal{D} \mid \boldsymbol{M})} .
$$

where $P\left(\mathcal{M}_{i} \mid \boldsymbol{M}\right)$ is the prior probability of each $\mathcal{M}_{i}$ and can be taken to be $1 / N_{M}$ if one considers all $N_{M}$ model classes are equally plausible; $p\left(\mathcal{D} \mid \mathcal{M}_{i}\right)$ expresses the probability of get- 
ting the data $\mathcal{D}$ based on $\mathcal{M}_{i}$ and is called evidence. It is given by the theorem of total probability:

$$
p\left(\mathcal{D} \mid \mathcal{M}_{i}\right)=\int p\left(\mathcal{D} \mid \boldsymbol{\theta}, \mathcal{M}_{i}\right) p\left(\boldsymbol{\theta} \mid \mathcal{M}_{i}\right) d \boldsymbol{\theta}
$$

It is worth noting that the log of evidence $p\left(\mathcal{D} \mid \mathcal{M}_{i}\right)$ can be expresses as the difference of two terms $[4,9]$ :

$$
\ln p\left(\mathcal{D} \mid \mathcal{M}_{i}\right)=\ln p\left(\mathcal{D} \mid \boldsymbol{\theta}, \mathcal{M}_{i}\right)-\ln \frac{p\left(\boldsymbol{\theta} \mid \mathcal{D}, \mathcal{M}_{i}\right)}{p\left(\boldsymbol{\theta} \mid \mathcal{M}_{i}\right)}
$$

The first term is the log of the likelihood function, which gives a measure of the goodness of the fit of the model class $\mathcal{M}_{i}$ to the data, and the second term is the Kullback-Leibler information, or relative entropy [10], which is a measure of the information gain about $\mathcal{M}_{i}$ from the data $\mathcal{D}$ and is always nonnegative. The importance of (22) is that it shows that the log evidence for $\mathcal{M}_{i}$, which controls the posterior probability of this model class according to (20), explicitly builds in a trade-off between the data-fit of the model class and its "complexity" (how much information it takes from the data).

In globally identifiable case [1], the posterior PDF for $\boldsymbol{\theta}$ given a large amount of data $\mathcal{D}$ may be approximated accurately by a Gaussian distribution, so $p\left(\mathcal{D} \mid \mathcal{M}_{i}\right)$ can be approximated by using Laplace's method for asymptotic approximation $[8,11]$ :

$$
p\left(\mathcal{D} \mid \mathcal{M}_{i}\right) \approx p\left(\mathcal{D} \mid \hat{\boldsymbol{\theta}}, \mathcal{M}_{i}\right) p\left(\hat{\boldsymbol{\theta}} \mid \mathcal{M}_{i}\right)(2 \pi)^{N_{i} / 2}\left|\mathbf{H}_{i}(\hat{\boldsymbol{\theta}})\right|^{-1 / 2}
$$

where $N_{i}$ is the number of uncertain parameters for the model class $\mathcal{M}_{i}$, the optimal parameter vector $\hat{\boldsymbol{\theta}}$ is the most probable value, and $\mathbf{H}_{i}(\hat{\boldsymbol{\theta}})$ is the Hessian matrix of $-\ln \left[p\left(\mathcal{D} \mid \boldsymbol{\theta}, \mathcal{M}_{i}\right) p\left(\boldsymbol{\theta} \mid \mathcal{M}_{i}\right)\right]$ with respect to $\boldsymbol{\theta}$ evaluated at $\hat{\boldsymbol{\theta}}$. Stochastic simulation methods $[9,12]$, can be used to evaluate the evidence when the Laplace asymptotic approximation for the evidence is not applicable, either because of an unidentifiable model class or because of computational difficulties in treating the inherent optimization problem in high dimensional parameter space.

In this study, model classes with different spatial resolution are considered to model the continuous medium for the update problem. The posterior probability of the candidate model classes is evaluated from (20) for comparison of the model classes.

\section{ILLUSTRATIVE EXAMPLE}

In this example, the identification of a soil-building system from simulated noisy total acceleration response data is considered (Figure 1). 500 four-node quad elements are used to model a $20 \mathrm{~m}$ tall soil column using the plane strain formulation of the quad element and the building is represented by a single degree of freedom (SDOF). The structure is assumed to be excited by a white noise base motion, which is not measured. The simulated dynamic data consists of total acceleration at the top of soil column and at the SDOF. The time interval used 
to generate the data is $0.01 \mathrm{sec}$ with total duration of $50 \mathrm{sec}$. The parameters used to generate the simulated data are: $\rho($ soil $)=2000 \mathrm{~kg} / \mathrm{m}^{3}, \quad E($ soil $)=50 \mathrm{MPa}$, Rayleigh damping $\zeta($ soil $)=0.05$ and SDOF with $f=1.6 \mathrm{~Hz}$ and $\zeta=0.02$. The variance of measured noise corresponds to $10 \%$ prediction error level, i.e. rms of the noise is $10 \%$ of the rms of the noise free response. The covariance matrix for the prediction-error $\mathbf{v}$ for the output vector equation is modeled as a diagonal matrix.

$$
\mathbf{R}=\sigma^{2} \mathbf{I}
$$

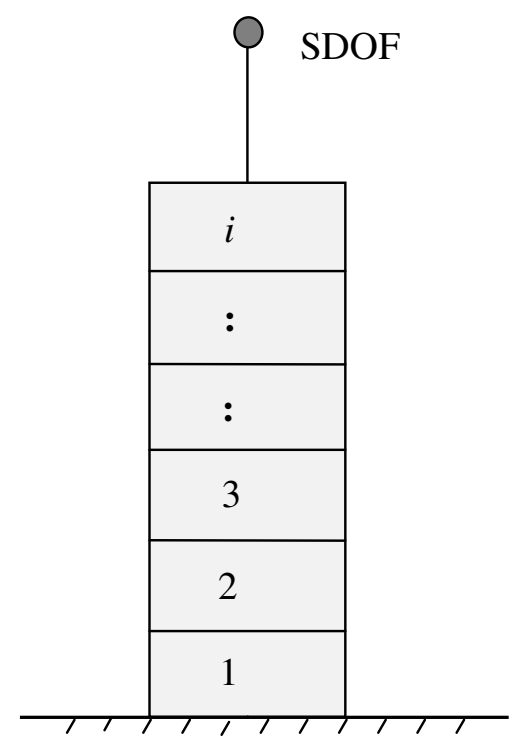

Figure 1. Soil-Building System.

In total, 6 parameters are considered to be uncertain that need to be identified: 2 soil parameters, 2 structural parameters and, the variances $Q$ and $\sigma^{2}, \boldsymbol{\theta}=\left[E_{\mathrm{s}}, \zeta_{\mathrm{s}}, f, \zeta, Q, \sigma^{2}\right]$. The likelihood of $\boldsymbol{\theta}$ can be obtained using equations (2)-(19). Non informative uniform prior distribution is assumed for all the uncertain parameters, $E=[25,100], \quad \zeta_{s}, \zeta=[0,0.2]$, $f=[0.8,3.2], Q, \sigma^{2}=[0,100]$. The posterior (updated) PDF of the uncertain system parameters is approximated by Gaussian distribution and Laplace asymptotic approximation is adopted to obtain the evidence.

A set of 10 model classes $\boldsymbol{M}=\left\{\mathcal{M}_{i}: i=5,10 . ., 50\right\}$ are considered where $\mathcal{M}_{i}$ is the model using $i$ elements to model the soil medium. Note that none of the model classes corresponds to the one used to generate the data. The goal here is to find the posterior probability of each model class given the dynamic data $\mathcal{D}$. All model classes are taken to be equally plausible, before getting any data from the system, i.e. $P\left(\mathcal{M}_{i} \mid \boldsymbol{M}\right)=1 / 10$.

Table 1 gives the optimal parameter values for some of the model classes and figure 23 shows the optimal soil stiffness and soil damping values for different model classes. As observed the optimal soil parameters are very close to the actual soil parameters and the structural (SDOF) parameters are very accurately identified for all the model classes. Figure 4 shows the value of $P\left(\mathcal{M}_{i} \mid \mathcal{D}, \boldsymbol{M}\right), \quad i=5,10 . ., 50$, calculated using equation (20). It is observed 
that the model with 5 soil elements is the most probable model and the contribution of higher modes to the response data is not significant enough to warrant their inclusion in making predictions of the system response. This conclusion needs to be further verified, however the result in this figure confirms the choice of spatial discretization scheme has significant effect on the parameter uncertainty especially those related to the soil properties.

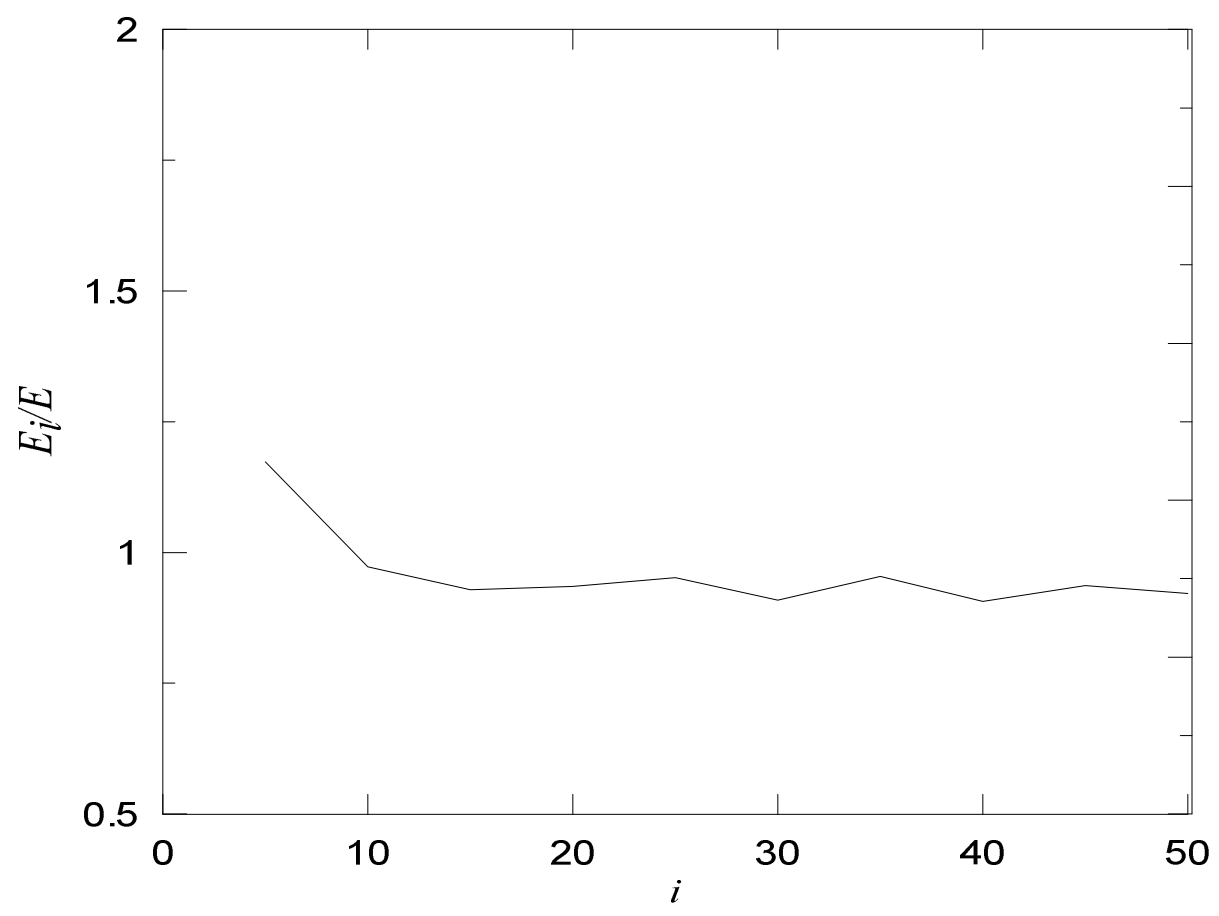

Figure 2. Optimal soil stiffness for different model classes.

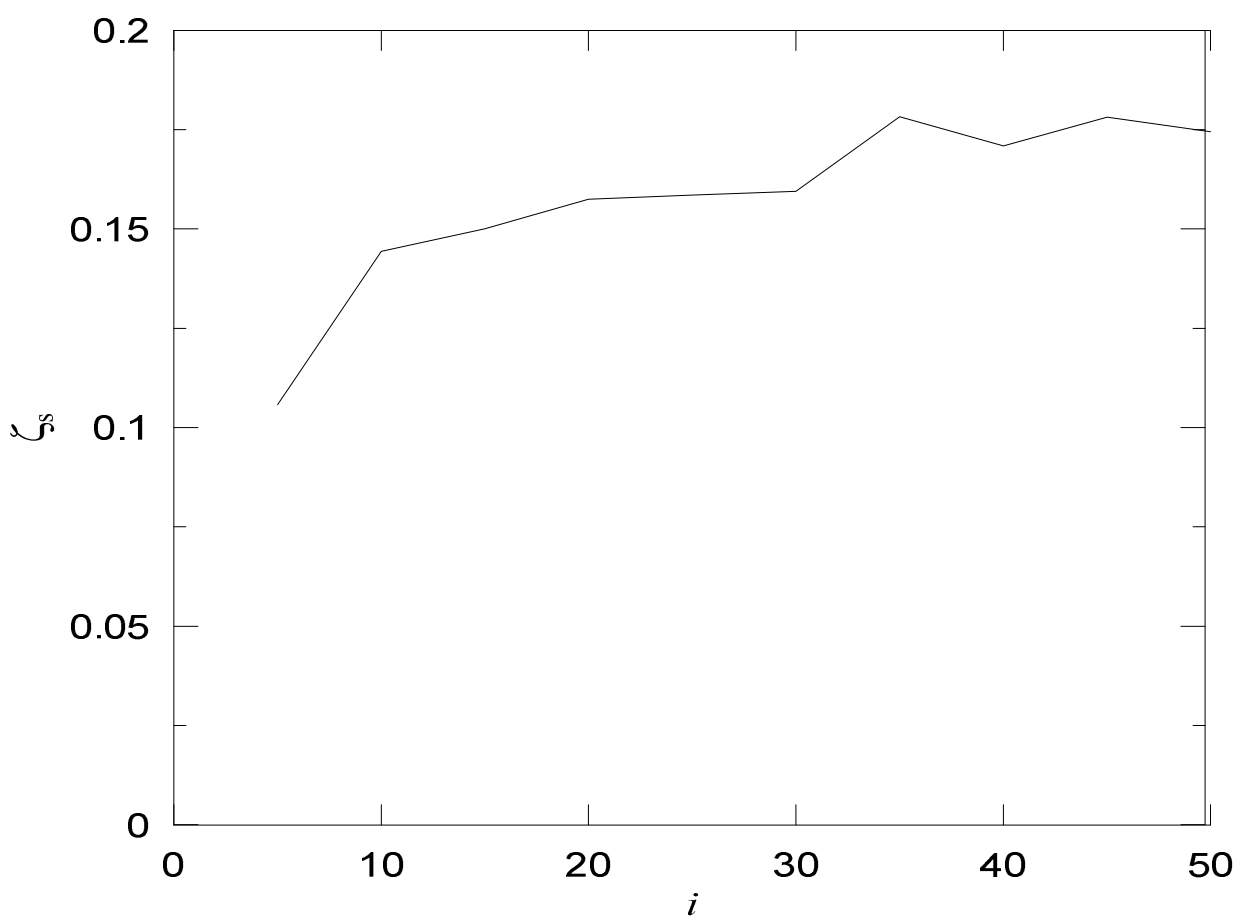

Figure 3. Optimal soil damping for different model classes. 


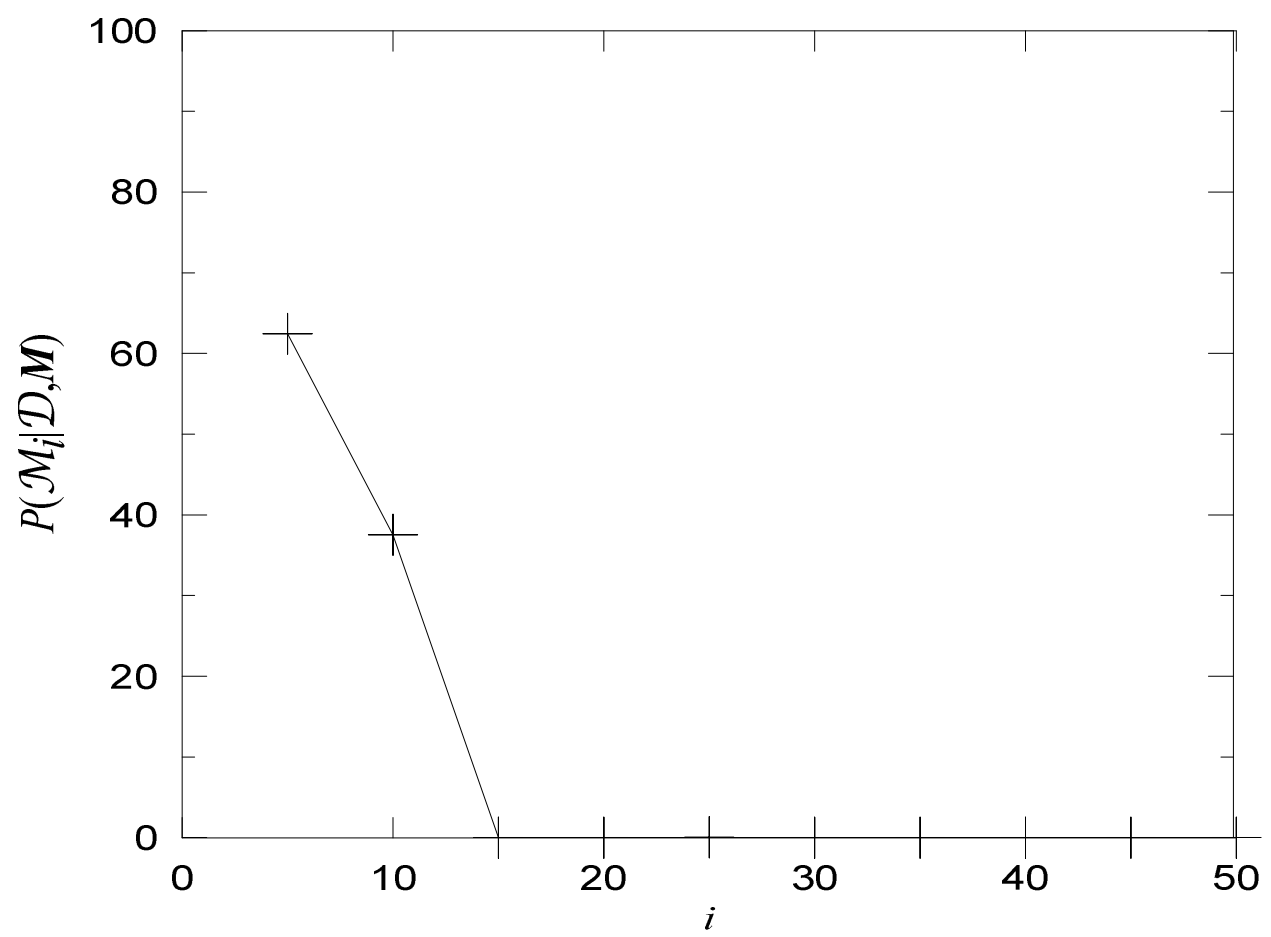

Figure 4. Probability of models with different number of soil elements based on data.

\section{CONCLUDING REMARKS}

Past application of the Bayesian model updating methodology focus on updating finite-element model parameters using any single spatial discretization scheme based on the measured data. In this paper, multiple model classes with different spatial discretization schemes are considered during the model updating. The results from the illustrative example show that the identified parameters using different discretization schemes can be quite different from each other, and model class consisting of a larger number of elements is not necessarily the more plausible one. The results are currently being obtained by another recently developed stochastic simulation algorithm that is more general and accurate than Laplace asymptotic approximation and will be presented in future publication.

Future focus will be on treating mesh discretization uncertain with continuous distribution, thus creating a single model class. Also, there is a need to study the effect of discretization scheme employed in the 2D and 3D spatial domain.

\section{REFERENCES}

[1] Beck, J.L., L.S. Katafygiotis, "Updating Models and Their Uncertainties. I: Bayesian Statistical Framework". Journal of Engineering Mechanics. 124(4), 455-461, 1998.

[2] Cheung, S.H., J.L. Beck, "Bayesian model updating using hybrid Monte Carlo simulation with application to structural dynamic models with many uncertain parameters". Journal of Engineering Mechanics. 135, 243, 2009. 
[3] Ching, J., M. Muto, J.L. Beck, "Structural model updating and health monitoring with incomplete modal data using Gibbs sampler". Computer Aided Civil and Infrastructure Engineering. 21(4), 242-257, 2006.

[4] Muto, M., J.L. Beck, "Bayesian Updating and Model Class Selection for Hysteretic Structural Models Using Stochastic Simulation". Journal of Vibration and Control. 14(1-2), 7-34, 2008.

[5] Papadimitriou, C., J.L. Beck, L.S. Katafygiotis, "Updating robust reliability using structural test data". Probabilistic Engineering Mechanics. 16(2), 103-113, 2001.

[6] Cheung, S.H., J.L. Beck. "Comparison of Different Model Classes for Bayesian Updating and Robust Predictions using Stochastic State-space System Models". Proc. 10th International Conference on Structural Safety and Reliability (ICOSSAR09). Osaka, Japan, 2009.

[7] Van Loan, C., "Computing integrals involving the matrix exponential". Automatic Control, IEEE Transactions on. 23(3), 395-404, 1978.

[8] Beck, J.L., K.V. Yuen, "Model Selection Using Response Measurements: Bayesian Probabilistic Approach". Journal of Engineering Mechanics. 130(2), 192-203, 2004.

[9] Cheung, S.H., J.L. Beck, "Calculation of posterior probabilities for Bayesian model class assessment and averaging from posterior samples based on dynamic system data". Computer-Aided Civil and Infrastructure Engineering. 25(5), 304-321, 2010.

[10] Cover, T.M., J.A. Thomas, J. Wiley, "Elements of information theory". Vol. 6: Wiley Online Library 1991.

[11] Papadimitriou, C., J.L. Beck, L.S. Katafygiotis, "Asymptotic Expansions for Reliability and Moments of Uncertain Systems". Journal of Engineering Mechanics. 123(12), 1219-1229, 1997.

[12] Ching, J., Y.C. Chen, "Transitional Markov Chain Monte Carlo Method for Bayesian Model Updating, Model Class Selection, and Model Averaging". Journal of Engineering Mechanics. 133(7), 816-832, 2007.

[13] Beck, J.L., S.K. Au, "Bayesian Updating of Structural Models and Reliability using Markov Chain Monte Carlo Simulation". Journal of Engineering Mechanics. 128(4), 380-391, 2002.

[14] Jaynes, E.T., "Probability Theory : The Logic of Science", Cambridge: Cambridge University Press. xxix, 727 p. 2003. 